\title{
Primary Localized Cutaneous Amyloidosis: A Retrospective Study of an Uncommon Skin Disease in the Largest Tertiary Care Center in Switzerland
}

\author{
Carole Guillet $^{a, b, c}$ Simona Steinmann ${ }^{a, c}$ Julia-Tatjana Maul ${ }^{a, c}$ Isabel Kolm ${ }^{a, c}$ \\ aDepartment of Dermatology, University Hospital Zurich, Zurich, Switzerland; bermatological Allergology, \\ Department of Dermatology and Allergy, Charité-Universitätsmedizin Berlin, corporate member of Freie Universität \\ Berlin, Humboldt-Universität zu Berlin, and Berlin Institute of Health, Berlin, Germany; ${ }^{\mathrm{C} F a c u l t y}$ of Medicine, \\ University of Zurich, Zurich, Switzerland
}

\section{Keywords}

Primary localized cutaneous amyloidosis · Lichen amyloidosis · Macular amyloidosis · Amyloid · Phototherapy · Topical corticosteroids

\begin{abstract}
Background: Primary localized cutaneous amyloidosis (PLCA) is defined by the deposition of amyloid protein in the skin without systemic involvement. There are four subtypes of PLCA: lichen amyloidosis (LA), macular amyloidosis (MA), biphasic amyloidosis (BA), and nodular amyloidosis (NA). PLCA occurs most frequently in Latin Americans and Asians. Treatment is not standardized. Objectives: To identify subtypes, demographic and clinical features and treatment efficacy in patients with histopathologically confirmed PLCA. Materials and Methods: Data of PLCA patients were extracted from the electronic hospital database and included if diagnosis of PLCA was histopathologically confirmed and if sufficient information regarding treatment and follow-up was available. The evaluation of the treatment efficacy was based on a novel score to assess the reduction of itch and skin lesions. Results: In this retrospective, monocentric
\end{abstract}

study, 37 cases of PLCA diagnosed between 2000 and 2020 were included ( 21 females) with a mean age of 52 years. LA was the most frequent subtype found in 21 patients (56.8\%), followed by MA in 10 patients (28\%) and BA in 6 patients (16.2\%). No cases of NA were included. 22 patients (59.4\%) had skin phototype II or III. Regarding treatment, a combination of UVA 1 phototherapy with high-potency topical corticosteroids seemed to show the highest efficacy with complete clearance of symptoms in 4 patients $(10.8 \%)$. A substantial improvement of symptoms was found in 5 patients (12.7\%) treated with high-potency topical corticosteroids alone or in combination either with UVA 1 or bath PUVA or monotherapy with UVA1 phototherapy or capsaicin $(0.075 \%)$ cream. Low-/medium-potency topical corticosteroids alone or in combination with UVBnb $(311 \mathrm{~nm})$ phototherapy showed a lower efficacy. Conclusion: Our data show that PCLA is a rare disease in central Europe but can also be expected in a predominantly Caucasian population. The best treatment response was achieved with a combination of UVA1 phototherapy and high-potency topical corticosteroids. (c) 2021 The Author(s)

Published by S. Karger AG, Basel karger@karger.com www.karger.com/drm

Karger $\stackrel{\text { ' }}{5}$

GOPEN ACCESS
(C) 2021 The Author(s)

Published by S. Karger AG, Basel

This is an Open Access article licensed under the Creative Commons Attribution-NonCommercial-4.0 International License (CC BY-NC) (http://www.karger.com/Services/OpenAccessLicense), applicable to the online version of the article only. Usage and distribution for commercial purposes requires written permission.
Correspondence to:

Carole Guillet, carole.guillet@usz.ch

Isabel Kolm, isabel.kolm-djamei@ usz.ch 


\section{Introduction}

Primary localized cutaneous amyloidosis (PLCA) is a rare skin disease characterized by the deposition of amyloid in the dermis without systemic involvement. Amyloid is an extracellular proteinaceous material which can accumulate in the skin and other organs. Amyloid in PLCA is derived from keratin peptides of necrotic keratinocytes and not, as in systemic forms of amyloidosis, from immunoglobulin or serum protein deposits [1]. The etiopathology of PLCA is not known. It has been hypothesized that signaling abnormalities via the oncostatin $\mathrm{M}$ type II receptor and/or the interleukin (IL)-31 receptor lead to keratinocyte apoptosis and subsequent amyloid deposition and pruritus $[2,3]$. Furthermore, point mutations in the IL-31 receptor A gene have been found in a hereditary form of PLCA [4]. It is assumed that the deposition of filamentous material secondary to intense scratching induced by pruritogenic processes leads to amyloid formation in the skin. Chronic scratching, as for example seen in atopic dermatitis, may promote damage to keratinocytes resulting in localized amyloid formation [5].

PLCA is thought to occur most frequently in individuals of Latin American or Asian origin [6]. Data on PLCA regarding demography, epidemiology, clinical patterns, and histopathological findings in Caucasians, however, is limited. Therefore, we retrospectively analyzed clinical patterns and treatment efficacy of PLCA in a tertiary care center in Switzerland.

\section{Subtypes and Clinical Manifestation}

There are four subtypes of PLCA: lichen amyloidosis (LA), macular amyloidosis (MA), and nodular amyloidosis (NA). A joint manifestation of LA and MA is called biphasic amyloidosis (BA) [7].

LA is the most frequent subtype of PLCA. It is characterized by multiple, localized or generalized, grouped papules with a predilection for the shins (Fig. 1). MA is characterized by multiple brown-greyish macules with a reticulated or rippled pattern and has a predilection for the upper back (Fig. 2). NA is the rarest form of PLCA and presents as firm pink to brown nodules preferentially involving the extremities. Nodule size varies from a few millimeters to centimeters and may coalesce to plaques $[8,9]$. Amyloid in NA is caused by light-chain amyloid synthesized by monoclonal plasma cells localized in the skin $[8,9]$. Previous studies have shown that up to $22 \%$ of patients with NA have underlying Sjörgren syndrome [10].

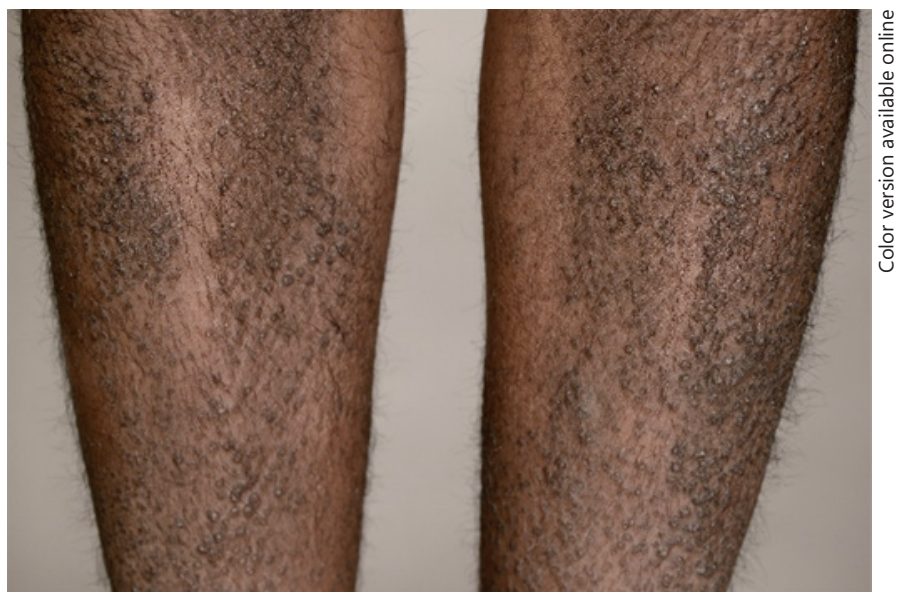

Fig. 1. Lichen amyloidosis manifesting as symmetrically distributed, hyperpigmented papules with central denting on both shins (male, 39 years, phototype V).

\section{Histopathology}

The key finding in PLCA is the deposition of pink, amorphous globular material in the papillary dermis. Additionally, increased pigmentation of the basal layer and scattered melanophages can be found. LA is characterized by marked acanthosis and hyperkeratosis. Typical histological findings of LA are shown in Figure 3.

For better visualization of the amyloid, special stains (congo red or pagoda) can be used (Fig. 4). Immunohistochemical staining with cytokeratin 5/6 (CK5/6) confirms the epidermal origin of the amyloid deposits in PLCA.

\section{Materials and Methods}

Patient Characteristics and Data Acquisition

After ethical approval (BASEC Nr.: 2019-02410), the electronic hospital database was searched for patients $\geq 18$ years old, treated at the Department of Dermatology at the University Hospital Zurich in the last 20 years (January 1, 2000, to December 31, 2020).

The following keywords were used to identify all patients diagnosed with PLCA: "Lichen amyloidosus," "lichen amyloidosis," "macular amyloidosis," "nodular amyloidosis," "primary cutaneous amyloidosis," "biphasic amyloidosis." Seventy-nine patients were identified using these criteria.

Out of these 79 patients, 42 patients had to be excluded from the present study because they did not meet the following inclusion criteria: (1) histologically confirmed diagnosis of PLCA, (2) availability of follow-up information for at least 3 months after diagnosis, (3) availability of information and photographic material regarding treatment response, (4) age $>18$ years (Fig. 5). In total, 9 patients with NA were identified. They either did not meet above-mentioned inclusion criteria or had concomitant mono- 


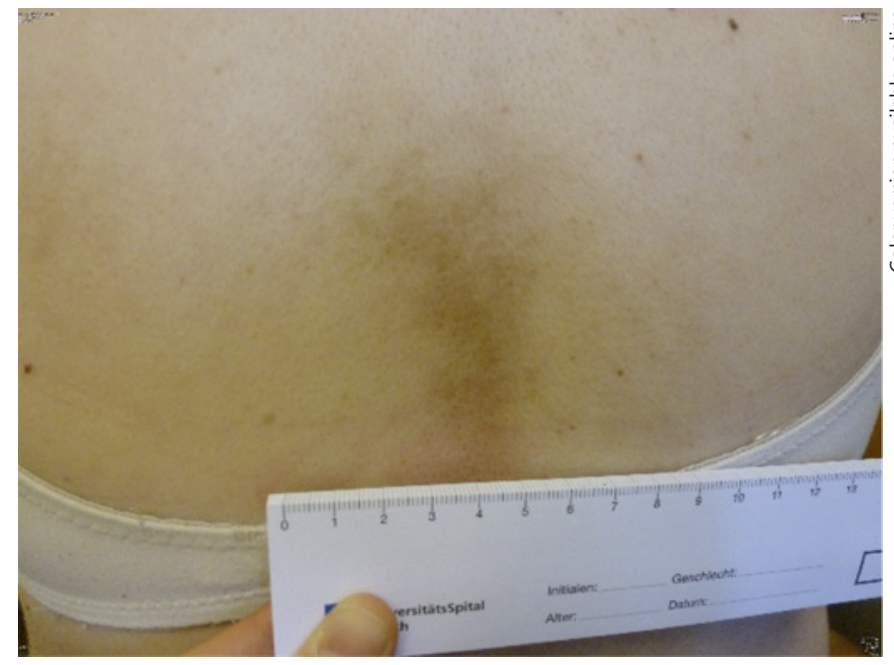

Fig. 2. Interscapular macular amyloidosis with hyperpigmentation (woman, 59 years, phototype III).

clonal gammopathy, and therefore amyloid deposits in the skin were interpreted as secondary to systemic disease. The following clinical information about the patients was extracted from the electronic database: age, sex, skin phototype (Fitzpatrick's scale I-VI), geographical region of origin, comorbidities, personal history of atopy, family history of PLCA, type of PLCA and treatment modalities (including treatment duration and treatment efficacy).

\section{Treatment Efficacy}

Treatment efficacy was assessed based on information available in the patients' medical records. To standardize treatment efficacy, we used a simple score to retrospectively assess treatment efficacy and reduction of itch: A score from 0 to 3 was applied to separately measure the itch and the reduction of skin changes including scaling, induration, hyperkeratosis, redness: $0=$ no effect on itch or skin, 1 = mild improvement of itch or skin, 2 = good improvement of itch or skin, 3 = very good improvement or complete resolution of itch or skin. The final score was calculated as the sum of both scores; a maximum score of 6 signified very good improvement or complete resolution of itch and skin changes. A minimal score 0 meant no treatment effect at all. The treatment efficacy score (TES) was established following a shared decision-making process by C.G. and I.K.

\section{Histopathology}

All histopathology specimens were reviewed by a board certified dermatopathologist (I.K.). Special stains (congo-red or pagoda stain) were performed in all cases; immunohistochemistry staining for CK5/6 was available in some cases.

\section{Statistical Analyses}

Descriptive analyses were performed using standard parameters (absolute and relative frequencies, means). Missing data were not imputed. Analyses were performed using IBM SPSS Statistics (IBM SPSS Statistics for Mac, Version 26.0; IBM Corp., Armonk, NY, USA).

PLCA - Clinical Characteristics and Treatment

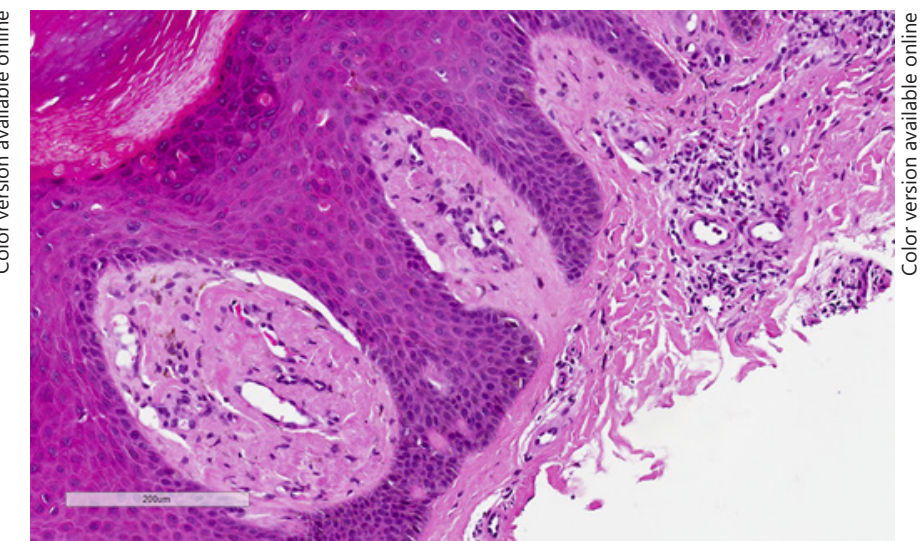

Fig. 3. Globular eosinophilic deposits (=amyloid) in the tips of the dermal papillae in LA. H\&E stain. $\times 400$.

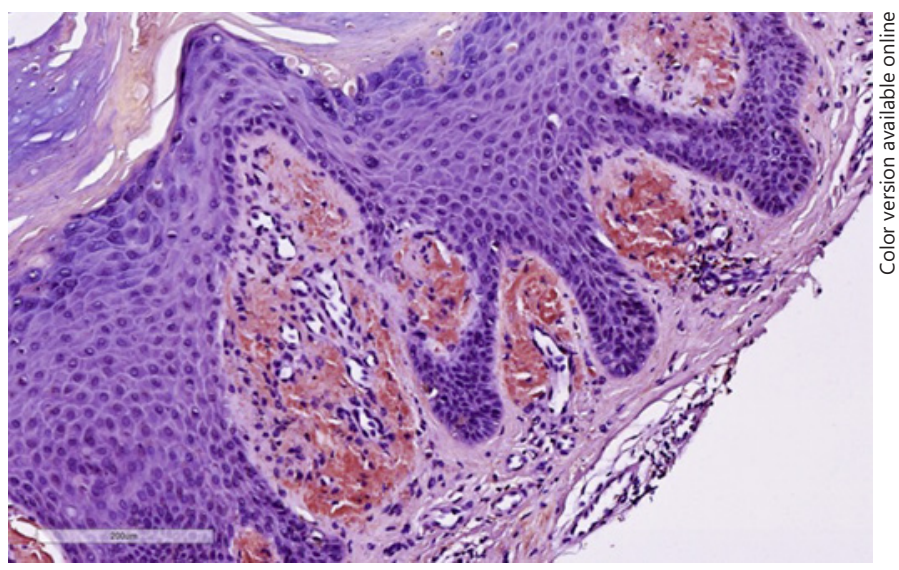

Fig. 4. Congo red stain highlighting globular orange-red deposits (=amyloid) in the tips of the dermal papillae in LA. $\times 400$.

\section{Results}

\section{Patient Characteristics}

A total of 37 patients with histopathologically confirmed PLCA and sufficient clinical information on follow-up and treatment were included in this study. Twenty-one patients (56.8\%) were female and 16 (43.2\%) male. The mean age was 52 years (range: $29-90$ years). Twentyone patients (56.8\%) presented with LA, 6 (16.2\%) with BA and 10 (27\%) with MA. Zero cases of NA were diagnosed. Eleven patients (29.7\%) were classified as skin phototype II, 11 patients (29.7\%) phototype III, 11 patients (29.7\%) phototype IV, and 4 patients (10.8\%) as phototype V. Skin phototype I and VI were not represented in the study.

Dermatology 2022;238:579-586 
Fig. 5. Flowchart of the methods: inclusion

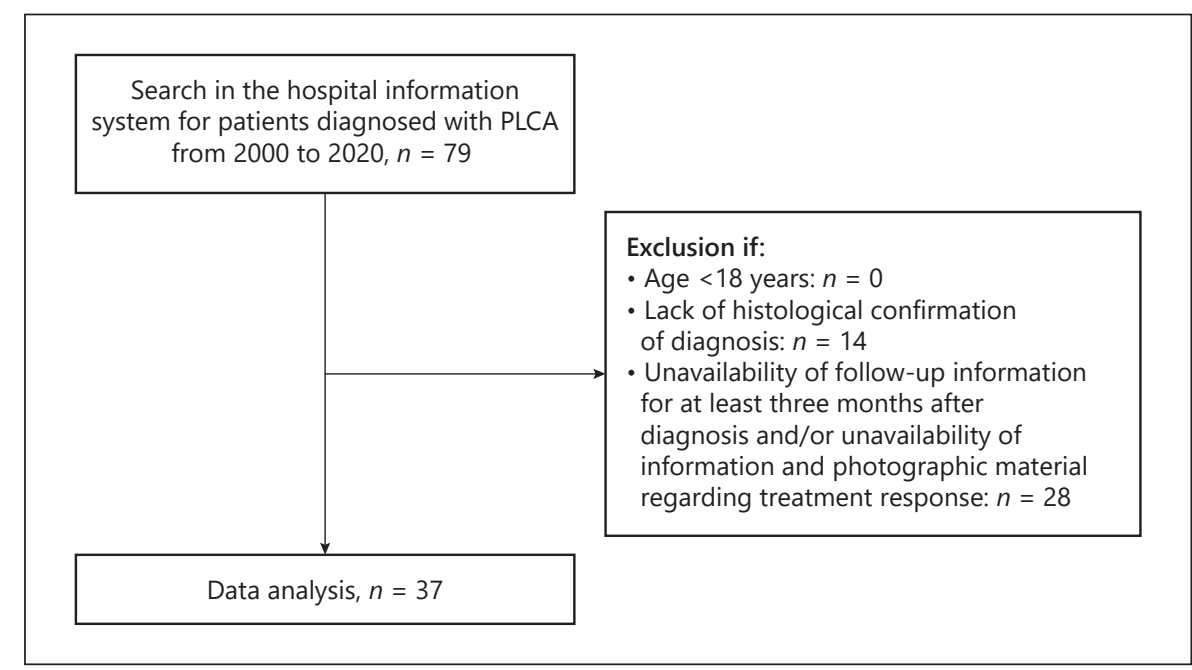
criteria.

Table 2. Treatment modalities $(n=37)$

\begin{tabular}{ll} 
Characteristics & \\
& \\
\hline Gender & \\
$\quad$ Female & \\
Male & $21(56.8)$ \\
Age, mean (range), years & $16(43.2)$ \\
Skin phototypes & $52(29-90)$ \\
I & \\
II & 0 \\
III & $11(29.7)$ \\
IV & $11(29.7)$ \\
V & $11(29.7)$ \\
VI & $4(10.8)$ \\
Clinical subtype & 0 \\
LA & \\
MA & $21(56.8)$ \\
BA & $10(27)$ \\
Localization & $6(16.2)$ \\
Lower legs & \\
Back/interscapular & $15(40.5)$ \\
Lower legs and arms & $15(40.5)$ \\
Mandibular & $2(5.4)$ \\
Lower arms & $2(5.4)$ \\
Abdomen & $1(2.7)$ \\
Generalized & $1(2.7)$ \\
Comorbidities & $1(2.7)$ \\
Atopy & \\
Thyroid disease & $8(21.6)$ \\
\hline
\end{tabular}

The regions of origin ("ethnicity") of all included patients were as follows: Central Europe: 15 patients (40.5\%); Mediterranean countries: 8 patients (21.6\%); China/ Southeast Asia/India/Sri Lanka/Mongolia: 10 patients (27\%); Middle East: 4 patients (10.8\%). Patients from cen-

\begin{tabular}{|c|c|}
\hline Treatment & $n(\%)$ \\
\hline \multicolumn{2}{|l|}{ Topical treatments } \\
\hline Low- to medium-potency topical corticosteroids & $16(43.2)$ \\
\hline High-potency topical corticosteroids & $7(18.9)$ \\
\hline Capsaicin $(0.075 \%)$ cream & $1(2.7)$ \\
\hline \multicolumn{2}{|l|}{ Phototherapy } \\
\hline UVBnb (311 nm) & $1(2.7)$ \\
\hline UVA1 & $2(5.4)$ \\
\hline Bath PUVA & $1(2.7)$ \\
\hline \multicolumn{2}{|l|}{ Combination treatments } \\
\hline $\begin{array}{l}\text { Low- to medium-potency topical corticosteroids } \\
\text { and UVBnb }\end{array}$ & $4(10.8)$ \\
\hline High-potency topical corticosteroids and UVA1 & $3(7.3)$ \\
\hline No treatment & $2(5.4)$ \\
\hline \multicolumn{2}{|c|}{$\begin{array}{l}\text { Treatment modalities were divided into topical treatments, pho } \\
\text { totherapy, and combination treatments. The largest number of pa } \\
\text { tients were treated with low- to medium-potency topical cortico } \\
\text { steroids. UVBnb, UVB narrow band phototherapy, wavelength: } 31 \\
\text { nm; UVA1, UVA1 phototherapy, wavelength: } 380 \mathrm{~nm} \text {; bath PUVA } \\
\text { whole body bath PUVA, wavelength: } 320-400 \mathrm{~nm} \text {. }\end{array}$} \\
\hline
\end{tabular}

tral Europe and Mediterranean countries were considered as Caucasian (23 patients, $61 \%$ ).

The most common localization of PLCA was on the lower legs $(15,40.5 \%)$ and back $(15,40.5 \%)$. Several comorbidities were noted: 8 patients $(21.6 \%)$ had a personal history of atopic eczema and 3 patients $(8.1 \%)$ had thyroid disease (two cases with hypothyroidism and one with hyperthyroidism of probable autoimmune origin). None of the patients had a positive family history for PLCA. Patient characteristics are summarized in Table 1. 
Table 3. Treatment efficacy (as measured by a treatment efficacy score - TES)

\begin{tabular}{lll}
\hline TES (0-6) & Patients, $n(\%)$ & Treatment modalities (number of patients) \\
\hline 0 & $2(5.4)$ & No treatment (2) \\
1 & $2(5.4)$ & TCS II-III (2) \\
2 & $11(29.7)$ & TCS II-III (6), TCS IV (2), UVA1 (1), UVBnb (1), UVBnb/TCSII (1) \\
3 & $5(12.7)$ & TCS II-III (2), TCS IV (2), UVBnb/TCSII (1) \\
4 & $8(21.6)$ & TCS II-III (5), TCS IV (2), UVBnb/TCSII (1) \\
5 & $5(12.7)$ & TCS IV (1), UVA1/TCSIV (1), PUVA (1), UVA1 (1), caps (1) \\
6 & $4(10.8)$ & TCS IV (1), UVA1/TCSIV (3) \\
\hline
\end{tabular}

A score from 0 to 3 was applied to separately measure the reduction of skin changes including scaling, induration, hyperkeratosis, redness AND of itch with $0=$ no effect, $1=$ mild improvement, $2=$ good improvement, $3=$ very good improvement or complete resolution. The final score was calculated as the sum of both scores; a maximum score of 6 signifies very good improvement or complete resolution of itch and skin changes. A minimal score 0 stands for no treatment effect at all. TCS II-II, mild to moderate topical corticosteroids; TCS IV, high-potency topical corticosteroids; UVA1/TCS IV, UVA1 and high-potency topical corticosteroids; UVBnb/TCS II, UVBnb and low- to medium-potency topical corticosteroids; PUVA, full body bath PUVA (320-400 nm); UVA1, UVA1 phototherapy (380 $\mathrm{nm})$; caps, capsaicin $0.075 \%$ cream; UVBnb, UVBnb phototherapy (311 nm).

\section{Treatment, Treatment Regimens and Treatment Efficacy}

Effect of treatment response was assessed in all patients after 3 months of treatment. Detailed information on treatment modalities and corresponding number of patients is given in Table 2. Most frequently, patients were treated with low- to medium-potency topical corticosteroids ( $n=16,43 \%$ ), followed by high-potency topical corticosteroids alone $(n=7,18.9 \%)$ and in combination with UVA1 phototherapy $(n=4,10.8 \%)$ or with a combination with low- to medium-potency topical corticosteroids and UVBnb phototherapy $(n=3,8.1 \%)$. Few patients were treated with phototherapy alone (UVA1: $n=2,5.4 \%$; UVBnb: $n=1,2.7 \%$ ), one patient received full body bath PUVA, and one patient was treated with topical capsaicin cream $(0.075 \%)$.

Treatment regimen of topical corticosteroids consisted in all cases of a once daily application for a maximum of 3 weeks and a subsequent tapering down of the application frequency over several weeks. Four patients received topical corticosteroids of moderate potency under occlusion using plastic film or occlusive dressings.

Medium-dose UVA1 (wavelength: $380 \mathrm{~nm}$ ), whole body bath PUVA (wavelength: 320-400 nm), and UVB narrowband (UVBnb) (wavelength: $311 \mathrm{~nm}$ ) were performed. UVA1 was initiated after phototesting on a small area and conducted at $50 \mathrm{~J} / \mathrm{cm}^{2}$. We performed 2-3 sessions per week for a maximum of 30 sessions, resulting in a maximal cumulative dose of $1,500 \mathrm{~J} / \mathrm{cm}^{2}$.

PLCA - Clinical Characteristics and Treatment
UVBnb phototherapy $(311 \mathrm{~nm})$ was started at $0.1 \mathrm{~J} /$ $\mathrm{cm}^{2}$ per session and increased by $20 \%$ from session to session. The maximum dose for UVBnb was $2.0 \mathrm{~J} / \mathrm{cm}^{2}$ and 2 to 3 sessions were held per week for a maximum of 12 weeks.

Bath PUVA was performed three times weekly for a duration of 12 weeks. The patient treated with capsaicin cream used a final concentration of capsaicin $0.075 \%$ cream three times daily on affected areas for a period of 12 weeks. All patients underwent a clinical re-evaluation after 12 weeks.

The maximum TES of 6 was achieved in four cases receiving high-potency topical corticosteroids in combination with UVA1 phototherapy $(n=3)$ or high-potency topical corticosteroids alone $(n=1)$. A submaximal score of 5 was achieved in 5 patients receiving high-potency topical corticosteroids $(n=1)$, UVA1 phototherapy $(n=$ $1)$, combination of high-potency topical corticosteroids and UVA 1 phototherapy $(n=1)$, PUVA therapy $(n=1)$ and capsaicin $(0.075 \%)$ cream $(n=1)$. TES of $2-4$ representing intermediate treatment responses was achieved by low- to medium-potency topical corticosteroids $(n=$ $13)$, by high-potency topical corticosteroids $(n=3)$, by a combination of topical corticosteroids and UVBnb phototherapy $(n=2)$ and by UVA 1 phototherapy $(n=1)$ and UVBnb phototherapy $(n=1)$, respectively. No treatment response (score: 0 ) and very mild treatment response (score: 1) was seen in 4 patients either not receiving any treatment at all $(n=2)$ or low- to medium-potency topical corticosteroids $(n=2)$. Table 3 summarizes the effi- 

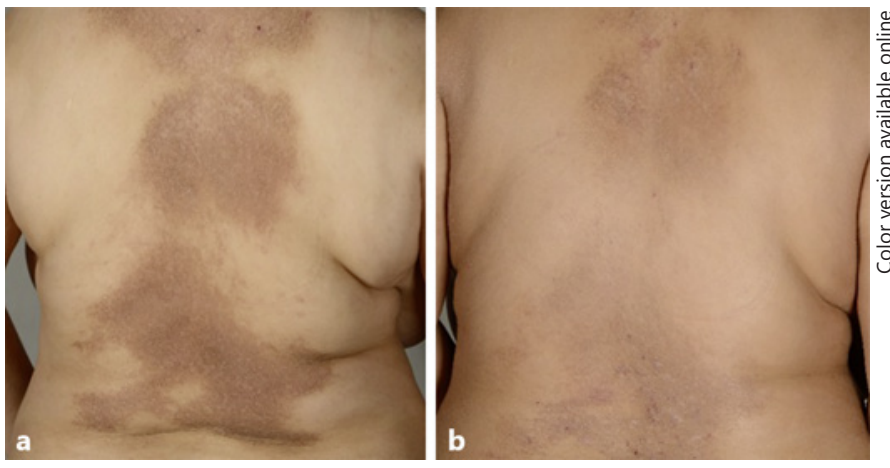

Fig. 6. a, b Interscapular, biphasic amyloidosis in a female patient (54 years, phototype IV) undergoing treatment with bath PUVA. Panel $\mathbf{a}$ shows the skin changes before and panel $\mathbf{b}$ shows the reduction of hyperpigmentation and erythema after 3 months of treatment. Bath PUVA had only minimal anti-pruritic effect in this patient.

cacy of the different treatment modalities. Figure 6 shows a patient with BA before and after treatment with bath PUVA for 3 months.

\section{Discussion}

The aim of this retrospective study was to analyze demographic characteristics and treatment outcomes of patients with PLCA in a tertiary care center in Switzerland. This is of relevance, since it is the first study on PLCA in a predominantly Caucasian population and analyzes the treatment efficacy in a larger group of patients. Knowledge on treatment modalities and efficacy in PLCA is very limited as there are only small studies and case reports on therapeutic strategies available.

In the dermatological outpatient department of the University Hospital Zurich, Switzerland, more than 22,000 patients are treated every year. Over a period of 20 years (2000-2020), we identified a total of 79 patients with PLCA, but only 37 patients met the inclusion criteria for this study. The low number of PCLA cases confirms that PLCA is a rare skin disease.

PLCA most frequently occurs in patients from Southeast Asia, China, and South America [11-13].

Interestingly, almost $60 \%$ of all patients in our study had skin phototype II or III and were of central European and Mediterranean origin. Our results underline that Caucasians with fairer skin can develop PLCA even though, worldwide, the disease is more common in darker phototypes and probably more expectable in non-Caucasian patients.
Our study did not show any gender differences and confirmed that PLCA usually affects middle-aged patients [8]. As expected, LA was the most common subtype of PLCA found in our study group [14]. Almost 30\% of our patients had either a history of atopic skin disease or of thyroid disease - a relationship of these pruritic diseases that has been well established [12, 15-20].

Chronic scratching is believed to lead to apoptosis of keratinocytes and consecutive amyloid deposition in the dermis [18-20]. Therefore, treatment should aim to reduce pruritus as much as possible, as amyloid formation is potentially the end-stage rather than the cause of the skin disease. We found that the best treatment responses were achieved with a combination of UVA1 phototherapy and a once daily application of high-potency topical corticosteroids.

The use of low- to medium-potency topical corticosteroids and/or UVBnb phototherapy and a series of other treatments seemed to show lower efficacy.

Topical corticosteroids reduce itch by inhibiting the release of pruritogenic pro-inflammatory cytokines [21]. Phototherapy is thought to modulate cutaneous sensory nerve fibers directly or by inducing mediator release from skin cells, reducing pruritus [22]. We suppose that UVA1 phototherapy led to better treatment outcomes in our patients than UVBnb phototherapy as longer wavelength light (UVA1) can penetrate deeper into the skin, which is frequently thickened and shows significant acanthosis and hyperkeratosis, especially in LA [23].

An explanation for the favorable treatment response on skin changes to the combination of topical corticosteroids and phototherapy might be the reduction in keratinocyte hyperproliferation, as has been shown in psoriasis [24-26]. As a consequence, combining the two treatment modalities can result in less thickening and hyperkeratosis of the epidermis, two of the typical clinical features of PLCA.

Our study has several limitations. An important limitation of our study is the retrospective, monocentric design, and the lack of a control cohort. Furthermore, the total number of patients is low and the number of patients with different treatments and treatment outcomes is high, which does not allow for robust statistics to be made. Forty out of 79 patients with suspected PLCA could not be included in this study due to the unavailability of followup information or a lack of histological confirmation of the diagnosis. One might suspect that patients with complete remission of symptoms or well-controlled disease were lost to follow-up, and therefore are not represented in this study. 
However, our data contribute to the increasing effort in defining appropriate treatment strategies in this group of rarer, benign, and noninfectious skin diseases. Highpotency topical corticosteroids in combination with UVA1 phototherapy seem to have a superior efficacy compared to mild- to medium-potency topical corticosteroids and UVBnb phototherapy. Further studies on larger patient cohorts and with prospective, randomized study designs will be needed to identify the best treatment options.

\section{Key Message}

Primary localized cutaneous amyloidosis is most effectively treated with high-potency topical corticosteroids and UVA1 phototherapy.

\section{Statement of Ethics}

This study was conducted ethically in accordance with the guidelines for human studies and in accordance with the World Medical Association Declaration of Helsinki.

The study protocol was approved by the Institute's committee on human research (Kantonale Ethik-Kommission Zürich, BASEC Nr.: 2019-02410).

Written informed consent was obtained from all participants if diagnosed after 2016. An exemption to retrospectively collect anonymized clinical data was made by abovementioned ethics committee if they diagnosis was made before 2016 and patients could not be contacted (BASEC Nr.: 2019-02410).

\section{Conflict of Interest Statement}

C.G. has served as advisor and/or received speaking fees and/ or participated in clinical trials sponsored by AbbVie, Almirall, Amgen, Celgene, Eli Lilly, Novartis. T.M. has served as advisor and/or received speaking fees and/or participated in clinical trials sponsored by AbbVie, Almirall, Amgen, BMS, Celgene, Eli Lilly, LEO Pharma, Janssen-Cilag, MSD, Novartis, Pfizer, Pierre Fabre, Roche, Sanofi, UCB. S.S., I.K.: no conflicts of interest to declare.

\section{Funding Sources}

C.G. received a research grant from EAACI (European Academy of Allergy and Clinical Immunology) to perform research outside of the submitted work at the Department of Dermatology of Charité - Universitätsmedizin, Berlin.

\section{Author Contributions}

C.G.: Conception and design, acquisition/analysis and interpretation, drafting of the manuscript, final approval, agreement to be accountable for all aspects of the work in ensuring that questions related to the accuracy or integrity of any part of the work are appropriately investigated and resolved. I.K.: Conception and design, data interpretation, drafting of the manuscript, final approval, Agreement to be accountable for all aspects of the work in ensuring that questions related to the accuracy or integrity of any part of the work are appropriately investigated and resolved. J.T.M.: Conception and design, data interpretation, critical revision for important intellectual content, final approval, agreement to be accountable for all aspects of the work in ensuring that questions related to the accuracy or integrity of any part of the work are appropriately investigated and resolved. S.S.: Conception and design, data interpretation, critical revision for important intellectual content, final approval, agreement to be accountable for all aspects of the work in ensuring that questions related to the accuracy or integrity of any part of the work are appropriately investigated and resolved.

\section{Data Availability Statement}

The raw data that support the findings of this study are available on request from the corresponding authors (C.G. or I.K.).

\section{References}

1 Breathnach SM. Amyloid and amyloidosis. J Am Acad Dermatol. 1988;18(1 Pt 1):1-16. Pt 1

2 Tanaka A, Arita K, Lai-Cheong JE, Palisson F, Hide M, McGrath JA. New insight into mechanisms of pruritus from molecular studies on familial primary localized cutaneous amyloidosis. Br J Dermatol. 2009;161:1217-24.

3 Tey HL, Yosipovitch G. Itch in ethnic populations. Acta Derm Venereol. 2010;90(3):227-34.

4 Lin MW, Lee DD, Liu TT, Lin YF, Chen SY, Huang CC, et al. Novel IL31RA gene mutation and ancestral OSMR mutant allele in familial primary cutaneous amyloidosis. Eur J Hum Genet. 2010;18(1):26-32.
5 Weyers W, Weyers I, Bonczkowitz M, DiazCascajo C, Schill WB. Lichen amyloidosus: a consequence of scratching. J Am Acad Dermatol. 1997;37:923-8.

6 Andrese E, Vâță D, Ciobanu D, Stătescu L, Solovăstru LG. The autoimmune constellation in lichen amyloidosis. Rev Med Chir Soc Med Nat Iasi. 2015 Oct-Dec;119(4):1045-50.

7 Jih MH. Biphasic cutaneous amyloidosis. Dermatol Online J. 2001;7(2):15.

8 Moon AO, Calamia KT, Walsh JS. Nodular amyloidosis: review and long-term follow-up of 16 cases. Arch Dermatol. 2003;139:1157-9.
9 Ritchie SA, Beachkofsky T, Schreml S, Gaspari A, Hivnor CM. Primary localized cutaneous nodular amyloidosis of the feet: a case report and review of the literature. Cutis. 2014; 93(2):89-94.

10 Shiman M, Ricotti C, Miteva M, Kerdel F, Romanelli P. Primary localized cutaneous nodular amyloidosis associated with CREST (calcinosis, Raynaud's phenomenon, esophageal motility disorders, sclerodactyly, and telangiectasia) syndrome. Int J Dermatol. 2010;49:229-30.

11 Tay CH, Dacosta JL. Lichen amyloidosis. Clinical study of 40 cases. Br J Dermatol. 1970; 82(2):129-36.
PLCA - Clinical Characteristics and Treatment 
12 Wong CK. Lichen amyloidosus. A relatively common skin disorder in Taiwan. Arch Dermatol. 1974;110(3):438-40.

13 Habermann MC, Montenegro MR. Primary cutaneous amyloidosis: clinical, laboratorial and histopathological study of 25 cases. Identification of gammaglobulins and C3 in the lesions by immunofluorescence. Dermatologica. 1980;160(4):240-8.

14 Weidner T, Illing T, Elsner P. Primary Localized Cutaneous Amyloidosis: A Systematic Treatment Review. Am J Clin Dermatol. 2017;18:629-42.

15 Shanon J. Cutaneous amyloidosis associated with atopic disorders. Dermatologica. 1970; 141(4):297-302.

16 Apaydin R, Bilen N, Bayramgürler D, Harova G, Cetinarslan B. Lichen amyloidosis, ankylosing spondylitis and autoimmune thyroiditis: coincidence or association? J Eur Acad Dermatol Venereol. 2000;14(2):135-7.
17 Dahdah MJ, Kurban M, Kibbi AG, Ghosn S. Primary localized cutaneous amyloidosis: a sign of immune dysregulation? Int J Dermatol. Apr 2009;48(4):419-21.

18 C T KL. Generalised pruritus as a presentation of Grave's disease. Malays Fam Physician. 2013 Apr 30;8(1):20-3. [not available in pubmed,crossref]

19 Humeda Y, Beasley J, Calder K. Clinical resolution of generalized lichen amyloidosis with dupilumab: a new alternative therapy. Dermatol Online J. 2020;26(12).

20 Gönül M, Cakmak SK, Kayaçatin S. Generalized lichen amyloidosis and hyperthyroidism: coincidence or association. Postepy Dermatol Alergol. 2013;30(4):265-7.
21 Elmariah SB, Lerner EA. Topical therapies for pruritus. Semin Cutan Med Surg. 2011;30(2): $118-26$

22 Legat FJ. The Antipruritic Effect of Phototherapy. Front Med (Lausanne). 2018;5:333.

23 Prasad S, Coias J, Chen HW, Jacobe H. Utilizing UVA-1 Phototherapy. Dermatol Clin. 2020;38(1):79-90.

24 Khoo BP, Tay YK, Goh CL. Calcipotriol ointment vs. betamethasone 17 -valerate ointment in the treatment of lichen amyloidosis. Int J Dermatol. 1999;38(7):539-41.

25 Uva L, Miguel D, Pinheiro C, Antunes J, Cruz D, Ferreira J, et al. Mechanisms of action of topical corticosteroids in psoriasis. Int J Endocrinol. 2012;2012:561018.

26 Benezeder T, Wolf P. Resolution of plaquetype psoriasis: what is left behind (and reinitiates the disease). Semin Immunopathol. 2019; 41(6):633-44.41 